\title{
Disordered Totally Asymmetric Simple Exclusion Process: Exact Results
}

\author{
Kiran M. Kolwankar1t and Alexander Punnoose ${ }^{2}$ t \\ ${ }^{1}$ Department of Mathematics, Indian Institute of Science, Bangalore, 560 012, India \\ ${ }^{2}$ Department of Physics, Indian Institute of Science, Bangalore, 560 012, India
}

(Version: July 15, 1998; printed April 2, 2022)

\begin{abstract}
We study the effect of quenched spatial disorder on the current-carrying steady states of the totally asymmetric simple exclusion process with spatially disordered jump rates. The exact analytical expressions for the steady-state weights, and the current are found for this model in one dimension. We demonstrate how these solutions can be exploited to study analytically the exact symmetries of the system. In particular, we prove that the magnitude of the steady-state current is left invariant when the direction of all the allowed particle jumps are reversed. Or equivalently, we prove that for any given filling and disorder configuration, particle-hole transformation is an exact symmetry that leaves the steady-state current invariant. This non-trivial symmetry was recently demonstrated in numerical simulations by Tripathy \& Burma (preprint cond-mat/9711302).
\end{abstract}

PACS numbers: 05.60.+w,05.50.+q,05.40.+j,02.50.Ey

Driven diffusive systems have been a subject of extensive studies in recent years [1]. A key ingredient of these systems is the presence of a driving field due to which the underlying dynamics do not generically obey detailed balance, leading to steady states with non-vanishing currents. In the absence of a unifying theme that encompasses our understanding of nonequilibrium phenomena, these systems offer a relatively simple framework within which systems far from thermal equilibrium may be studied.

Driven diffusive systems further provide the possibility of studying the intriguing interplay of disorder, interactions and drive for a wide range of parameter values like the degree of disorder, filling and drive strength [2]. These expectations are not unfounded: (i) Driven diffusive systems in the absence of disorder have been studied extensively and have revealed basic differences between equilibrium and nonequilibrium systems [1, 3,, []. For instance, it has been demonstrated that spontaneous symmetry breaking (SSB) [5]6] and phase separation can occur in one dimensional asymmetric exclusion processes [7]. In contrast it is well known that one dimensional systems in thermal equilibrium with short range interactions do not exhibit phenomena like SSB and phase separation [8]; (ii) Also, systems with disorder and drive but no interactions between particles are well studied and understood 9].

A number of physical situations involving flow in random media require an understanding of disorder driven diffusive systems of interacting particles [10]. Most of the understanding gained in these system have been largely based on numerical simulations. Analytical characterization in terms of the exact steady state measures in systems without translational invariance have been found only in the case of the disordered drop-push model [11].

An interesting class of models for which there exists no analytical characterization of the steady-state weights or the steady-state current is the disordered totally asymmetric simple exclusion process (DTASEP). Even the single disorder case has not been amenable to an analytic treatment. Extensive numerical and mean-field studies have been done and a number of interesting features have been highlighted 11 13].

In this work we derive, for the first time, formally exact solutions for the steady-state weights, and current for the DTASEP model in one dimension. These solutions are valid for arbitrary disorder and particle fillings. We further show how these solutions can be exploited to study the exact symmetries of the model by studying the transformations that leave the magnitude of the steady-state current invariant. In particular we show that the model possesses an exact particle-hole symmetry for any given disorder realization; a result that was observed in numerical simulations by Tripathy \& Barma [14]. In addition we prove the "obvious" result that the steady-state current is a constant across each bond. Although this result is intuitively obvious, given that the dynamics conserves the number of particles, to the best of the authors knowledge no explicit proof of this statement for a disordered lattice model exists.

Definition of the model: The DTASEP model is defined on a 1D lattice of length $L$ with periodic boundary conditions. Each site can hold either 1 or 0 particle. Each bond $(i, i+1)$ of the lattice is assigned a quenched random rate $\alpha_{i}$ chosen independently from some chosen probability distribution. The evolution is governed by random sequential dynamics defined as follows: in a time interval $d t$ the particle attempts to hop, with probability $\alpha_{i} d t$ to its neighboring site $i+1$. We consider the case in which the jumps are allowed only in one direction (to the right) and is the same for all bonds. In addition, the move is completed if and only if site $i+1$ is unoccupied. 
The time averaged steady-state current $J_{i}$ in the bond $(i, i+1)$ is given by:

$$
J_{i}=\alpha_{i}\left\langle n_{i}\left(1-n_{i+1}\right)\right\rangle
$$

where $n_{i}$ counts the number of particles at site $i$.

Since for every particle hopping to the right, a "hole" jumps to the left, implies interchanging all the particles for holes (charge conjugation - C) and reversing the direction of hopping (time reversal - T) leaves the steady state current, up to a sign, the same. Hence the steadystate current is symmetric under a CT transformation. This symmetry is valid in general: in any dimension; in the presence of disorder; and in the case when the particle can hop along any direction with finite probabilities (DASEP).

In Eq. (1), if all the $\alpha_{i}$ 's are put equal to the same constant $\alpha$, then it follows that the steady state current $J_{0}$, which is assumed to be the same on each bond and hence $J_{0}=L^{-1} \sum_{i} J_{i}$, is invariant under $n_{i} \rightarrow 1-n_{i}$ for all $i$. Hence for the clean totally asymmetric simple exclusion process in 1D (TASEP), charge conjugation (C) in itself leaves the steady-state current invariant. Since CT symmetry is always true, it follows that for the TASEP model in 1D both charge conjugation and time reversal (T) symmetries individually holds.

The surprising observation made in the numerical simulations in Ref. [14] was that, charge conjugation symmetry was found to hold even in the presence of disorder in the DTASEP model in 1D. This symmetry as is obvious from Eq. (11) is not expected to hold when all the $\alpha_{i}$ 's are taken to be random. Convincing numerical evidence has been offered, although a general proof of the validity of this symmetry for all fillings and disorder configurations has not been obtained.

We derive below for this model a formally exact expression for the steady-state probability density and the current $J_{0}$. Using these expressions we show that the observed reflection symmetry holds in general for all fillings and disorder configuration which in turn implies the symmetry of the steady-state current under charge conjugation.

For $L$ sites with $N$ particles, the number of configurations $M={ }^{L} C_{N}$. Here ${ }^{L} C_{N}=L ! /(L-N) ! N !$ is the number of ways $N$ particles can be distributed amongst $L$ sites with a maximum of only one particle per site. The dynamics of these configurations, for a given realization of the quenched bond variables $\mathbf{R}=\left\{\alpha_{1}, \alpha_{2}, \cdots, \alpha_{L}\right\}$, are given by the rate equation:

$$
\frac{d P_{m}}{d t}=\sum_{n} T(n \rightarrow m) P_{n}-\sum_{n^{\prime}} T\left(m \rightarrow n^{\prime}\right) P_{m}
$$

where $T(m \rightarrow n)$ gives the transition probability from configuration $m \rightarrow n$. This expression can be conveniently expressed in matrix notation as:

$$
\frac{d \mathbf{P}(t)}{d t}=W \mathbf{P}(t)
$$

with $W_{m n}=T(n \rightarrow m)$ and $W_{m m}=-\sum_{n^{\prime}} T\left(m \rightarrow n^{\prime}\right)$. Since $\mathbf{1} \cdot \mathbf{P}(t)=\sum_{m} P_{m}(t)=1 \forall t$ (normalization), it follows that $\mathbf{1} \cdot W=0$. This implies that $W$ has a zero eigen-value with left eigen-vector $\mathbf{1}$. Hence there also exists a right eigen-vector $\mathbf{P}$ such that $W \mathbf{P}=0$. This defines the steady-state solution of the problem for a given choice of the disorder $\mathbf{R}$.

The steady-state of the problem considered above is characterized by a uniform current $J_{0}$ across each bond. Given the solution $\mathbf{P}$, the current across, say, site $i$ and $i+1$, is given as $\alpha_{i} \sum P_{m}=J_{0}$, where the sum is only over the set of configurations with a particle on site $i$ and a hole on site $i+1$ (see Eq. I).

The above sum for all sites $i$ can be combined and conveniently written in matrix notation by defining a matrix $G$ such that $G \cdot \mathbf{P}=J_{0}\left(1 / \alpha_{1}, 1 / \alpha_{2}, \cdots, 1 / \alpha_{L}\right)^{T} \equiv$ $J_{0}(1 / \alpha)^{T}$. The entries of $G$ for a given row $i$ (corresponding to site $i$ ) has 1 corresponding to the configurations with a particle at site $i$ and a hole at site $i+1$ and 0 otherwise. The order of the $G$ matrix is therefore $(L \times M)$ since there are $L$ sites and $M$ configurations. The $G$ matrix can be expanded to an $(M \times M)$ matrix by augmenting an $(M-L) \times M$ zero matrix to $G$, such that:

$$
Q \cdot \mathbf{P} \equiv\left(\begin{array}{l}
G_{\mathrm{L} \times \mathrm{M}} \\
0_{(\mathrm{M}-\mathrm{L}) \times \mathrm{M}}
\end{array}\right) \cdot \mathbf{P}=J_{0}\left(\begin{array}{c}
1 / \alpha \\
0
\end{array}\right) \equiv J_{0} \mathbf{V}
$$

where for convenience we have defined new variables $Q=(G, 0)^{T}$ and $\mathbf{V}=(1 / \alpha, 0)^{T}$. Hence the three equations that determine the steady-state distribution and current are:

$$
\begin{aligned}
W \cdot \mathbf{P} & =0 \\
Q \cdot \mathbf{P} & =J_{0} \mathbf{V} \\
\mathbf{1} \cdot \mathbf{P} & =1
\end{aligned}
$$

If we assume that the $\operatorname{row}-\operatorname{rank}(W)=M-1$ and not lesser, then there exists a one-parameter solution to Eq. (5a). The most general form of the solution will be of the form $\beta \mathbf{P}$, where $\beta$ is the arbitrary parameter. The solution when substituted into Eq. (bb) allows the $\beta$ factor to be absorbed in the definition of $J_{0}$ by re-scaling $J_{0} \rightarrow J_{0} / \beta$. Hence, the most general solution $\mathbf{P} \equiv \mathbf{P}\left(J_{0}\right)$ will have $J_{0}$ appearing as the only free parameter. The value of $J_{0}$ is fixed by the normalization condition given in Eq. (5d). Hence a unique $\mathbf{P}$ and $J_{0}$ solves Eq. (5).

It should be noted that both Eq. $5 \mathrm{a}$ and Eq. $5 \mathrm{~b}$ cannot be inverted to obtain a solution for $\mathbf{P}$ and $J_{0}$ as neither $W$ nor $Q$ are invertible matrices. Although, $W$ and $Q$ are independently non-invertible, their sum $Q+W$ is invertible. The proof of this is simple. Suppose $\operatorname{row}-\operatorname{rank}(Q+W)=r<M$. Then the equation: 


$$
(Q+W) \cdot \mathbf{P}=J_{0} \mathbf{V}
$$

obtained by adding Eqs. (5a) and (5b) would have a general solution $\mathbf{P}\left(J_{0}, \beta_{1}, \beta_{2}, \cdots, \beta_{M-r}\right)$, i.e., in addition to the free parameter $J_{0}$, there will be $M-r$ other free parameters. However, it was shown earlier that the most general solution of $\mathbf{P} \equiv \mathbf{P}\left(J_{0}\right)$ can have only one free parameter $J_{0}$, that can be fixed by normalization. It follows that $r=M$ which implies that $(Q+W)$ is invertible.

Using Eq. (6), we obtain $\mathbf{P}\left(J_{0}\right)=J_{0}(Q+W)^{-1} \cdot \mathbf{V}$. The steady-state current $J_{0}$ is obtained using the normalization condition in Eq. (5c) giving, $1 / J_{0}=\mathbf{1} \cdot(Q+W)^{-1} \cdot \mathbf{V}$. Hence the final solutions are given as:

$$
\begin{aligned}
\mathbf{P} & =\frac{(Q+W)^{-1} \cdot \mathbf{V}}{\mathbf{1} \cdot(Q+W)^{-1} \cdot \mathbf{V}} \\
J_{0} & =\frac{1}{\mathbf{1} \cdot(Q+W)^{-1} \cdot \mathbf{V}}
\end{aligned}
$$

Note that in general a linear combination:

$$
(Q+\eta W) \cdot \mathbf{P}=J_{0} \mathbf{V}
$$

could have been used. This is equivalent to the equation $(Q+W) \cdot \mathbf{P}+(\eta-1) W \cdot \mathbf{P}=J_{0} \mathbf{V}$. Since $\mathbf{W} \cdot \mathbf{P}=0$ we get back Eq. (6) implying that $\mathbf{P}$ will be independent of $\eta$ as it should be.

In arriving at the above solutions, we had assumed that in steady-state, the current across each bond was the same constant $J_{0}$. The arguments presented above clearly indicate that there would be no unique solution had we started by assuming that the currents were different. For suppose the currents across the bonds were $\left\{J_{1}, J_{2}, \cdots, J_{L}\right\}$, then the most general solution would be of the form $\mathbf{P}\left(J_{1}, J_{2}, \cdots, J_{L}\right)$. Since there are $L$ unknowns but only one normalization condition, a unique solution would not exist. We therefore conclude that the currents in steady state are a constant across each bond.

We now use these solutions to prove the reflection symmetry of the current observed in Ref. [14]. It was, as stated earlier, observed in Monte Carlo simulations that, for a given realization of the quenched bond variables $\mathbf{R}=\left\{\alpha_{1}, \alpha_{2}, \cdots, \alpha_{L}\right\}$, the steady state-current was the same whether the particles were allowed to jump to the right or to the left.

We denote the set of quenched random bond variables when the particles are allowed to jump to the left as $\overline{\mathbf{R}}=\left\{\alpha_{1}, \alpha_{2}, \cdots, \alpha_{L}\right\}$. In $\overline{\mathbf{R}}$ the magnitude and the ordering of the $\alpha_{i}$ 's are the same as in $\mathbf{R}$ although the direction of hopping has been reversed. Let $\overline{\mathbf{P}}$ be the steadystate weights and $\bar{W}$ and $\bar{Q}$ the corresponding matrices such that $\bar{W} \cdot \overline{\mathbf{P}}=0$ and $\bar{Q} \cdot \overline{\mathbf{P}}=\bar{J}_{0} \mathbf{V}$. The $\mathbf{V}$ vector in both cases are the same and $\bar{J}_{0}$ is the appropriate current.

The question we ask here is: Does there exist an invertible matrix $S$, such that:

$$
\begin{aligned}
Q \cdot S & =\bar{Q} \\
S^{-1} \cdot \mathbf{P} & =\overline{\mathbf{P}}
\end{aligned}
$$

The existence of such an $S$ would imply that $\bar{J}_{0}=J_{0}$, since:

$$
\bar{Q} \cdot \overline{\mathbf{P}}=Q \cdot S S^{-1} \cdot \mathbf{P}=Q \cdot \mathbf{P}
$$

We first derive the properties that $S$ should satisfy. Since each row of $G$ contains as many 1's as the number of configuration for which there exists a particle at site $i$ and a hole at site $i+1$, we have $G \cdot \mathbf{1}={ }^{L-2} C_{N-1} \mathbf{1}$. The same is true of $\bar{G}$, although the exact location of each of the 1's will differ since the direction of hopping is reversed. Going back to Eq. (9a), we get:

$$
Q \cdot S\left(\begin{array}{l}
\mathbf{1} \\
\mathbf{1}
\end{array}\right)=\bar{Q}\left(\begin{array}{l}
\mathbf{1} \\
\mathbf{1}
\end{array}\right)={ }^{L-2} C_{N-1}\left(\begin{array}{l}
\mathbf{1} \\
\mathbf{0}
\end{array}\right)=Q\left(\begin{array}{l}
\mathbf{1} \\
\mathbf{1}
\end{array}\right)
$$

All the conditions in the above equation can be satisfied if we take $S \cdot \mathbf{1}=\mathbf{1}$

We now analyze Eq. (9b) to put further constraints on $S$. Since both $\mathbf{P}$ and $\overline{\mathbf{P}}$ are normalized, we obtain $\mathbf{1} \cdot \mathbf{P}=\mathbf{1} \cdot S \cdot \overline{\mathbf{P}}=1$ This equation can be satisfied if we take $\mathbf{1} \cdot S=\mathbf{1}+\mathbf{A}$, with the additional constraint that $\mathbf{A} \cdot \overline{\mathbf{P}}=0$. We observe that the equation is satisfied only up to a vector $\mathbf{A}$ which is 'orthogonal' to $\overline{\mathbf{P}}$.

Acting by $W$ on both sides of Eq. (9b), gives:

$$
W \cdot \mathbf{P}=W S \cdot \overline{\mathbf{P}}=0
$$

This equation can be satisfied if we take $W S= \pm \bar{W}$. The sign can be fixed by observing that $W S \cdot \mathbf{1}=W \cdot \mathbf{1}=$ $\pm \bar{W} \cdot \mathbf{1}$. Where we have used the property that $S \cdot \mathbf{1}=\mathbf{1}$. Since the direction of hopping has been reversed in $\overline{\mathbf{R}}$, the outgoing configurations w.r.t. a given initial particle configuration are mapped to the incoming configurations in $\mathbf{R}$ and vice versa, we obtain $W \cdot \mathbf{1}=-\bar{W} \cdot \mathbf{1}$ (refer to Eqs. (2\& 3)).

Thus the two equations that $S$ should satisfy are:

$$
\begin{aligned}
Q S & =\bar{Q} \\
W S & =-\bar{W}
\end{aligned}
$$

An important feature to be noted is that it is not a similarity transformation that relates the two systems defined by $W$ and $\bar{W}$. By adding the two equations, we obtain:

$$
S=(Q+W)^{-1} \cdot(\bar{Q}-\bar{W})
$$

Choosing $\eta=-1$ in Eq. (8) and $\overline{\mathbf{P}}=J_{0}(\bar{Q}-\bar{W})^{-1} \cdot \mathbf{V}$ with $1 / J_{0}=\mathbf{1} \cdot(\bar{Q}-\bar{W})^{-1} \cdot \mathbf{V}$ from Eq. $(\overline{7})$, it can be shown that $S$ indeed satisfies Eq. (9). This completes our proof that the steady-state currents for $\mathbf{R}$ and $\overline{\mathbf{R}}$ are the same.

In conclusion, following an algebraic procedure, we have derived formally exact expressions for the steadystate probability density $\mathbf{P}$ and the current $J_{0}$ for the 
totally asymmetric disordered simple exclusion process in one dimension. We explicitly show how these solutions can be exploited to study exact symmetries that leave the steady-state current invariant. In particular we have demonstrated that the steady-state current, up to a sign, is the same when the direction of allowed jumps is reversed. We also explicitly show that in steady-state the current across each bond is the same constant, a result intuitively obvious but lacking proof for disordered lattice models. Although not showed explicitly here, the choice of $S$ in Eq. (9) is a special case of the more general requirement that $Q \cdot \mathbf{P}=\overline{\mathbf{Q}} \cdot \overline{\mathbf{P}}$, which guarantees the currents to be the same under a symmetry operation. Hence, we believe that the methodology we have developed has wider applicablility in studying and understanding the symmetries of more general models not amenable to direct analytical studies.

On completion of this work, we were made aware of an alternate proof of the time reversal symmetry of the DTASEP model given in Ref. 15 which in contrast to our algebraic proof is probabilistic in character.

We would like to thank Mustansir Barma and Goutam Tripathy for bringing this problem to our notice and a careful reading of the manuscript. We thank Drs. S. Goldstein and E. Speer for sharing their unpublished results with us. One of us (AP) acknowledges very fruitful discussions with Chandan Das Gupta, Toby Joseph and Amit Puniyani and KMK is greatful to the Department of Science and Technology, India, (DST/PAM/GR/381) for financial assistance.
* kirkol@math.iisc.ernet.in

† alex@physics.iisc.ernet.in

[1] B. Schmittmann and R. K. P. Zia, in Phase Transitions and Critical Phenomena, edited by C. Domb and J. L. Lebowitz (Academic Press, London, 1995), Vol. 17.

[2] D. S. Fisher, in Nonlinearity in Condensed Matter, edited by A. R. Bishop, D. K. Campbell, P. Kumar and S. E. Trullinger (Springer-Verlag, Berlin, 1987).

[3] F. Spitzer, Adv. Math., 5, 246 (1970).

[4] T. M. Liggett, Interacting Particle Systems (Springer, NY, 1985).

[5] M. R. Evans, D. P. Foster, C. Godrèche and D. Mukamel, Phys. Rev. Lett., 74, 208 (1995).

[6] C. Godrèche et al., J. Phys. A, 28, 6039 (1995).

[7] R. Lahiri and S. Ramaswamy, Phys. Rev. Lett., 79, 1150 (1997).

[8] L. D. Landau and E. M. Lifshitz, Statistical Physics I (Pergammon, Oxford 1980).

[9] J. -P. Bouchaud and A. Georges, Phys. Rep. 195, 127 (1990).

[10] O. Narayan and D. S. Fisher, Phys. Rev. B, 49, 9469 (1994).

[11] G. Tripathy and M. Barma, Phys. Rev. Lett., 78, 3039 (1997).

[12] S. A. Janowsky and J. L. Lebowitz, Phys. Rev. A, 45, 618 (1992).

[13] G. Schütz, J. Stat. Phys., 75, 471 (1993).

[14] G. Tripathy and M. Barma, preprint (1997, condmat/9711302).

[15] S. Goldstein and E. R. Speer (private communications) 